\title{
Interfacial elastic fingering in Hele-Shaw cells: A weakly nonlinear study
}

\author{
Gabriel D. Carvalho and José A. Miranda* \\ Departamento de Física, Universidade Federal de Pernambuco, Recife, Pernambuco 50670-901, Brazil
}

Hermes Gadêlha

Wolfson Centre for Mathematical Biology, Mathematical Institute, University of Oxford, Oxford OX2 6GG, United Kingdom and Department of Applied Mathematics and Theoretical Physics, University of Cambridge, Cambridge CB3 OWA, United Kingdom

(Received 17 September 2013; published 11 November 2013)

\begin{abstract}
We study a variant of the classic viscous fingering instability in Hele-Shaw cells where the interface separating the fluids is elastic, and presents a curvature-dependent bending rigidity. By employing a second-order modecoupling approach we investigate how the elastic nature of the interface influences the morphology of emerging interfacial patterns. This is done by focusing our attention on a conventionally stable situation in which the fluids involved have the same viscosity. In this framework, we show that the inclusion of nonlinear effects plays a crucial role in inducing sizable interfacial instabilities, as well as in determining the ultimate shape of the pattern-forming structures. Particularly, we have found that the emergence of either narrow or wide fingers can be regulated by tuning a rigidity fraction parameter. Our weakly nonlinear findings reinforce the importance of the so-called curvature weakening effect, which favors the development of fingers in regions of lower rigidity.
\end{abstract}

DOI: 10.1103/PhysRevE.88.053006

PACS number(s): 47.15.gp, 47.70.Fw, 47.54.-r, 47.20.Ma

\section{INTRODUCTION}

The celebrated Saffman-Taylor instability [1] occurs when a viscous fluid is injected into another fluid of higher viscosity in the confined geometry of a Hele-Shaw cell [2]. Under such circumstances, the fluid-fluid interface is unstable leading to the growth of fingerlike shapes. For radial fluid injection [3-9] the fingers tend to multiply and evolve through repeated tipsplitting, ultimately forming complex branched patterns. In the context of the classic Saffman-Taylor problem there is no instability when a more viscous fluid displaces a less viscous one, constituting a stable, reverse flow displacement. In this case the fluid-fluid interface propagates in the form of a stable circular front. Conventional Hele-Shaw flow with viscositymatched fluids is also stable.

In some situations of practical and technological interest one might need to favor the development of interfacial instabilities, even during reverse or viscosity-matched flows. For example, it is known the formation of interfacial instabilities is quite advantageous to improve mixing processes in small-scale devices [10]. In fact, there has been recent increased interest in trying to trigger the instability in conventionally stable Hele-Shaw flow conditions. Very recent studies $[11,12]$ have demonstrated, by linear stability analysis and experiments, that the introduction of a small gradient in the gap of the Hele-Shaw cell (so that the cell plates are no longer parallel) can induce the Saffman-Taylor instability under classically stable situations [13].

Other suggestive strategies have been proposed to destabilize the fluid-fluid interface in confined Hele-Shaw flows. The development of the reversed viscous fingering instability has been investigated both theoretically and experimentally by considering the addition of surfactants to the moving interface, or to the Hele-Shaw cell plates [14-20]. The presence of the surfactant may cause the surface tension to vary along

\footnotetext{
*jme@df.ufpe.br
}

the fluid-fluid boundary, facilitating the occurrence of local disturbances. Another line of study examines the appearance of chemohydrodynamic instabilities when chemical reactions and diffusion interplay with advection or convection processes [21-26]. Sophisticated numerical simulations have detected the occurrence of a reversed Saffman-Taylor instability in miscible viscous fingering due to double-diffusive effects [23]. Laboratory experiments of reaction-driven miscible viscous fingering $[24,25]$ confirmed that chemical reactions can indeed destabilize the classically stable reverse situation.

A particularly interesting experiment involving the occurrence of interfacial chemical reactions in Hele-Shaw geometry has been performed by Podgorski et al. in Ref. [27], who performed experiments using two miscible fluids of equal viscosities. The miscible fluids react at the interface, producing a gel-like phase of increased stiffness. This system turned out to be unstable, leading to the formation of a variety of interfacial patterns, including mushroom-shaped structures and tentacle-like fingers.

A subsequent theoretical investigation tried to mimic the reactive miscible system examined in [27], by considering that the two fluids are immiscible, but that the interface separating them is an elastic membrane, presenting a curvaturedependent bending rigidity [28]. They have assumed that the gel-like phase at the interface prevents further mixing, so that miscibility plays a negligible role in determining the fingering instability. In this scenario, they have considered a modified Young-Laplace pressure jump boundary condition that contemplates the fact that the interface has a bending rigidity changing according to the local interfacial curvature. Their linear stability results have been able to account for the fact that the reactive system is more unstable than the nonreactive one, so that reaction has a destabilizing effect. In particular, it has been shown that the interface can become unstable even if the fluids have the same viscosity, consistently with the experimental findings of Ref. [27].

Despite the significance of the linear stability results obtained in Ref. [28] regarding the stability of the interface, at 


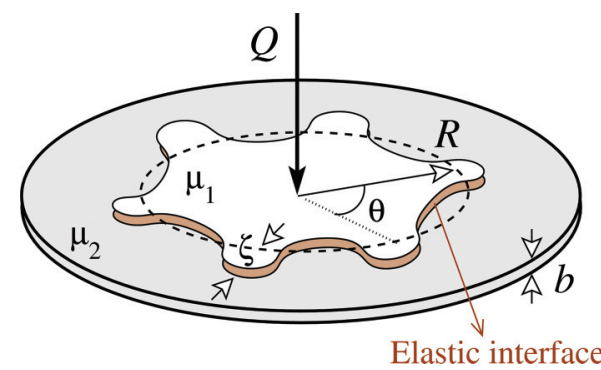

FIG. 1. (Color online) Representative sketch of a radial HeleShaw cell with an elastic interface separating fluids 1 and 2.

purely linear stages not much can be said about the influence of the elastic interface on the morphology of the emergent fingering patterns. To properly address this type of effect one must investigate the nonlinear stages of the dynamics. This is precisely what we do in this work: by using the theoretical model proposed in Ref. [28] as a starting point, we employ a second-order mode-coupling perturbative scheme [8] to get important insights into the onset of pattern formation processes in this interfacial elastic fingering instability problem.

\section{WEAKLY NONLINEAR SCHEME}

Consider a Hele-Shaw cell of gap spacing $b$ containing two immiscible, incompressible, viscous fluids (see Fig. 1). Denote the viscosities of the inner and outer fluids, respectively, as $\mu_{1}$ and $\mu_{2}$. Fluid 1 is injected into fluid 2 at a constant injection rate $Q$ (equal to the area covered per unit time). Due to a chemical reaction there exists a gel-like interface separating the two fluids. As in Refs. [28,29] we treat the interface as an elastic membrane, presenting a curvature-dependent bending rigidity.

The perturbed fluid-fluid interface is described as $\mathfrak{R}(\theta, t)=$ $R(t)+\zeta(\theta, t)$, where $\theta$ represents the azimuthal angle, and $R(t)$ is the time dependent unperturbed radius $R=R(t)=$ $\sqrt{R_{0}^{2}+Q t / \pi}$, with $R_{0}$ being the unperturbed radius at $t=0$. Here, $\zeta(\theta, t)=\sum_{n=-\infty}^{+\infty} \zeta_{n}(t) \exp (\operatorname{in} \theta)$ denotes the net interface perturbation with Fourier amplitudes $\zeta_{n}(t)$, and discrete wave numbers $n$. Our perturbative approach keeps terms up to the second order in $\zeta$. In the Fourier expansion of $\zeta$ we include the $n=0$ mode to maintain the area of the perturbed shape independent of the perturbation $\zeta$. Mass conservation imposes that the zeroth mode is written in terms of the other modes as $\zeta_{0}=-(1 / 2 R) \sum_{n \neq 0}\left|\zeta_{n}(t)\right|^{2}$.

For the effectively two-dimensional geometry of the radial Hele-Shaw cell, the governing equation of the system is the gap-averaged Darcy's law [3,27]

$$
\mathbf{v}_{j}=-\frac{b^{2}}{12 \mu_{j}} \nabla p_{j}
$$

where $\mathbf{v}_{j}$ and $p_{j}$ denote the velocity and pressure in fluids $j=1,2$, respectively. From the irrotational nature of the flow $\left(\nabla \times \mathbf{v}_{j}=0\right)$, and the incompressibility condition

$$
\nabla \cdot \mathbf{v}_{j}=0,
$$

it can be readily verified that the velocity potential $\phi_{j}\left(\mathbf{v}_{j}=\right.$ $-\nabla \phi_{j}$ ) obeys Laplace's equation. To get the equation of motion for the interface, we rewrite Eq. (1) for each of the fluids in terms of the velocity potential. Integrate and then subtract the resulting equations from each other to obtain [8]

$$
A\left(\frac{\phi_{1}+\phi_{2}}{2}\right)-\left(\frac{\phi_{1}-\phi_{2}}{2}\right)=-\frac{b^{2} \Delta p}{12\left(\mu_{1}+\mu_{2}\right)},
$$

where the dimensionless parameter $A=\left(\mu_{2}-\mu_{1}\right) /\left(\mu_{2}+\mu_{1}\right)$ is the viscosity contrast, and

$$
\Delta p=\left.\left(p_{1}-p_{2}\right)\right|_{r=\mathfrak{R}}-\left.\left(p_{1}-p_{2}\right)\right|_{r=R},
$$

where $\left.\left(p_{1}-p_{2}\right)\right|_{r=\Re}$ denotes the pressure jump on the perturbed interface, while $\left.\left(p_{1}-p_{2}\right)\right|_{r=R}$ represents the pressure jump on the unperturbed interface [28,29].

To include the contributions coming from the elastic nature of the fluid-fluid interface, similarly to what was done in [28,29] we consider a generalized Young-Laplace pressure boundary condition, which expresses the pressure jump across the perturbed fluid-fluid interface as

$$
\begin{aligned}
\left.\left(p_{1}-p_{2}\right)\right|_{r=\Re}= & -\frac{1}{2} v^{\prime \prime \prime} \kappa^{2} \kappa_{s}^{2}-v^{\prime \prime}\left(3 \kappa \kappa_{s}^{2}+\frac{1}{2} \kappa^{2} \kappa_{s s}\right) \\
& -v^{\prime}\left(\frac{1}{2} \kappa^{4}+3 \kappa_{s}^{2}+2 \kappa \kappa_{s s}\right)-v\left(\frac{1}{2} \kappa^{3}+\kappa_{s s}\right),
\end{aligned}
$$

where

$$
v=v(\kappa)=v_{0}\left[C e^{-\lambda^{2} \kappa^{2}}+1-C\right]
$$

is the bending rigidity function, $v_{0}$ is the maximum rigidity that expresses the largest resistance to disturbances, and $0 \leqslant$ $C<1$ is the rigidity fraction, which measures the fraction of intramolecular bonds broken through surface deformation. Note that the constant rigidity limit is reached in Eq. (6) by setting $C=0 . \kappa$ denotes the interface curvature in the plane of the cell, and $\lambda>0$ is a characteristic radius [28]. In Eq. (5) the primes indicate derivatives with respect to the curvature $\kappa$, while the subscripts of $\kappa$ indicate derivatives with respect to the arc length $s$. The details of our computation leading to Eq. (5) are presented in Appendix A. Our calculation of Eq. (5) is distinct from the one presented in Ref. [29] but we obtain the same final result.

The problem is specified by the generalized pressure jump boundary condition (5), plus the kinematic boundary condition which states that the normal components of each fluid's velocity are continuous at the interface [2]

$$
\frac{\partial \Re}{\partial t}=\left(\frac{1}{r^{2}} \frac{\partial \Re}{\partial \theta} \frac{\partial \phi_{i}}{\partial \theta}\right)_{r=\Re}-\left(\frac{\partial \phi_{i}}{\partial r}\right)_{r=\mathfrak{R}} .
$$

Following standard steps performed in previous weakly nonlinear studies for radial Hele-Shaw flows [8,20], first we define Fourier expansions for the velocity potentials. Then, we express $\phi_{j}$ in terms of the perturbation amplitudes $\zeta_{n}$ by considering condition (7). Substituting these relations, and the pressure jump condition Eq. (5) into Eq. (3), always keeping terms up to second order in $\zeta$, and Fourier transforming, we find the equation of motion for the perturbation amplitudes (for $n \neq 0$ )

$$
\dot{\zeta}_{n}=\Lambda(n) \zeta_{n}+\sum_{m \neq 0}\left[F(n, m) \zeta_{m} \zeta_{n-m}+G(n, m) \dot{\zeta}_{m} \zeta_{n-m}\right]
$$


where the overdot denotes total time derivative,

$$
\begin{aligned}
\Lambda(n)= & \frac{Q}{2 \pi R^{2}}(A|n|-1)+\frac{\alpha \nu_{0}}{2 R^{5}}|n|\left(n^{2}-1\right) \\
& \times\left[A_{1}(C, \eta)\left(n^{2}+1\right)+A_{2}(C, \eta)\right]
\end{aligned}
$$

is the linear growth rate,

$$
\begin{gathered}
A_{1}(C, \eta)=C e^{-\eta}\left(-4 \eta^{2}+10 \eta-2\right)-2(1-C), \\
A_{2}(C, \eta)=C e^{-\eta}\left(8 \eta^{2}-22 \eta+5\right)+5(1-C),
\end{gathered}
$$

with $\alpha=b^{2} /\left[12\left(\mu_{1}+\mu_{2}\right)\right]$, and $\eta=(\lambda / R)^{2}$.

The second-order mode-coupling terms are given by

$$
\begin{aligned}
F(n, m)= & \frac{|n|}{R}\left\{\frac{Q A}{2 \pi R^{2}}\left[\frac{1}{2}-\operatorname{sgn}(n m)\right]-\frac{\alpha v_{0} C e^{-\eta}}{R^{5}}\right. \\
& \times\left[B_{1}(n, m)+\eta B_{2}(n, m)+\eta^{2} B_{3}(n, m)\right. \\
& \left.\left.+2 \eta^{3} B_{4}(n, m)\right]-\frac{\alpha v_{0}(1-C)}{R^{5}} B_{1}(n, m)\right\},
\end{aligned}
$$

and

$$
G(n, m)=\frac{1}{R}\{A|n|[1-\operatorname{sgn}(n m)]-1\},
$$

where the sgn function equals \pm 1 according to the sign of its argument. The expressions for the functions $B_{1}(n, m)$, $B_{2}(n, m), B_{3}(n, m)$, and $B_{4}(n, m)$ are given in Appendix B.

Equations (8)-(13) represent the mode-coupling equations of the elastic fingering instability problem in a radial HeleShaw cell, considering that the interface has a curvaturedependent bending rigidity. This set of nonlinear equations opens up the possibility of investigating analytically how the morphology of the evolving interface respond to the interplay between injection, and the own elastic features of the fluid-fluid boundary.

At the linear level the interplay mentioned above is clearly manifested by the linear growth rate (9): the first term is related to the effect of injection while the second term expresses the bending rigidity contribution. For a given $n$, in the limit of constant rigidity $(C=0)$ the quantity $\left[A_{1}(C, \eta)\left(n^{2}+1\right)+A_{2}(C, \eta)\right]$ is negative, and the bending forces stabilize the interface. On the other hand, considering injection $Q>0$ (and not suction, where $Q<0$ ), it can either destabilize or stabilize the system depending on the sign of the viscosity contrast $(-1 \leqslant A \leqslant+1)$. However, if $0<$ $C<1$ the quantity $\left[A_{1}(C, \eta)\left(n^{2}+1\right)+A_{2}(C, \eta)\right]$ can become positive and the effect of the curvature-dependent bending may lead to interface destabilization. In this work, we focus on the important situation in which $A=0$ (viscosity-matched fluids as in Ref. [27]), $0<C<1$, and $Q>0$ so that the injection contribution is stabilizing, and bending forces are destabilizing.

\section{ONSET OF NONLINEARITIES}

In this section we use our mode-coupling approach to investigate the interface evolution at first and second order. We illustrate the usefulness of our weakly nonlinear analysis in elucidating key aspects related to shape of emergent fingers during the development of the interfacial elastic instability in radial Hele-Shaw cells. To simplify our discussion it is convenient to rewrite the net perturbation in terms of cosine and sine modes

$$
\zeta(\theta, t)=\zeta_{0}+\sum_{n=1}^{\infty}\left[a_{n}(t) \cos (n \theta)+b_{n}(t) \sin (n \theta)\right],
$$

where $a_{n}=\zeta_{n}+\zeta_{-n}$ and $b_{n}=i\left(\zeta_{n}-\zeta_{-n}\right)$ are real-valued. Without loss of generality we may choose the phase of the fundamental mode so that $a_{n}>0$ and $b_{n}=0$.

We stress that the values we take for our parameters throughout this work are consistent with typical physical quantities used in real experiments for conventional injectiondriven radial viscous flows in Hele-Shaw cells [2-7], and with related experimental and theoretical studies involving the development of the elastic fingering instability in this flow geometry [27-29]. We consider fluids of equal viscosities $\left[\mu_{1}=\mu_{2}=0.3 \mathrm{~g} /(\mathrm{cm} \mathrm{s})\right]$, so that we have a zero viscosity contrast situation $(A=0)$. The constant injection rate is taken as $Q=0.85 \mathrm{~cm}^{2} / \mathrm{s}$, and the thickness of the cell $b=0.06 \mathrm{~cm}$. The initial radius $R_{0}$ is of the order of $1.0 \mathrm{~cm}$, and the evolution of the interfaces we consider typically run up to a few seconds time. Specific values for $R_{0}$ and time $t$ will be provided in the discussion of each different situation examined in the remainder of this work. Finally, unless otherwise stated we take $C=0.99, \lambda=1.0 \mathrm{~cm}$, and $\nu_{0}=0.8 \mathrm{~g} \mathrm{~cm}^{2} / \mathrm{s}^{2}$.

As commented in Sec. I, the reactive miscible fingering experiments performed by Podgorski et al. [27] exhibited a number of finger shapes. Generally speaking, depending on the flow parameters one could get tentacle-like fingers or mushroom-shaped structures. While the convoluted tentaclelike patterns are characterized by thin fingers with narrow tips, the mushroom-shaped structures present swollen fingering protrusions with wide, inflated tips. Of course, these intricate patterns are highly nonlinear and their complex shapes could not be accurately reproduced by our weakly nonlinear Fourier analysis. However, we can use our mode-coupling approach to try to detect the onset of such nonlinear effects, and then get analytical insight into the tendency toward finger tip-widening or finger tip-narrowing already at early nonlinear stages of the dynamics. In addition, this can be conveniently done by utilizing just a small number of interacting Fourier modes [8].

Within our mode-coupling approach the finger tip behavior is related to the influence of a fundamental mode $n$ on the growth of its harmonic $2 n$ [8]. Under these circumstances, the equations of motion for the cosine and sine modes of the harmonic are

$$
\begin{gathered}
\dot{a}_{2 n}=\Lambda(2 n) a_{2 n}+\frac{1}{2} T(2 n, n) a_{n}^{2}, \\
\dot{b}_{2 n}=\Lambda(2 n) b_{2 n},
\end{gathered}
$$

where

$$
T(n, m)=F(n, m)+\Lambda(m) G(n, m) .
$$

From Eq. (16) we can see that the growth of the sine mode $b_{2 n}$ is uninfluenced by $a_{n}$, and does not present second-order couplings, so we focus on the growth of the cosine modes. Likewise, the equation of motion for the fundamental mode is

$$
\dot{a}_{n}=\Lambda(n) a_{n}+\frac{1}{2}[T(n, 2 n)+T(n,-n)] a_{n} a_{2 n} .
$$


(a)

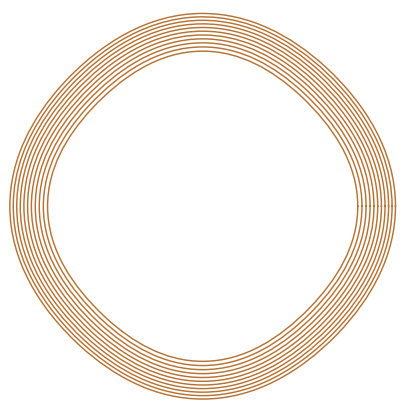

(c)

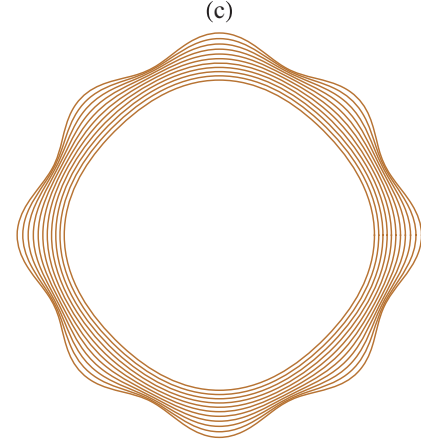

(b)

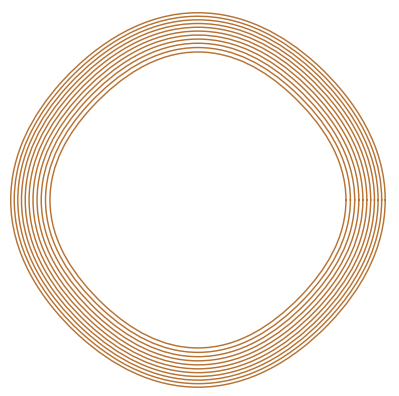

(d)

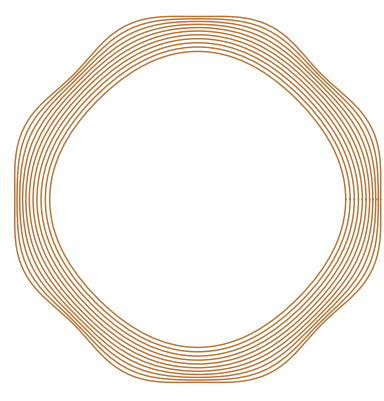

FIG. 2. (Color online) Snapshots of the evolving interface for the interaction of two cosine modes $n=4$ and $2 n=8$. Purely linear evolution for (a) $R_{0}=1.2 \mathrm{~cm}$, and (b) $R_{0}=1.145 \mathrm{~cm}$. Weakly nonlinear evolution for (c) $R_{0}=1.2 \mathrm{~cm}$, and (d) $R_{0}=1.145 \mathrm{~cm}$. Recall that here $C=0.99$.

In fact, Eq. (15) shows that the presence of the fundamental mode $n$ forces growth of the harmonic mode $2 n$. It has been shown in Ref. [8] that if $a_{2 n}$ is driven negative, one observes finger tip-widening, and even finger tip-splitting. On the other hand, if $a_{2 n}$ is driven positive finger tip-narrowing results. Next, we study how these finger tip mechanisms act in the presence of an elastic fluid-fluid interface.

To examine the influence of the elastic interface effects on finger tip behavior at second order, in Fig. 2 we plot the time evolution of the cosine perturbation amplitudes for the fundamental mode $a_{n}$, and for its harmonic mode $a_{2 n}$. This is done by solving the coupled nonlinear Eqs. (15) and (18). Figures 2(a) and 2(b) show the purely linear evolution of the interface for initial radii $R_{0}=1.2 \mathrm{~cm}$, and $R_{0}=1.145 \mathrm{~cm}$, respectively. On the other hand, Figs. 2(c) and 2(d) depict the weakly nonlinear evolution of the interface, again for initial radii $R_{0}=1.2 \mathrm{~cm}$, and $R_{0}=1.145 \mathrm{~cm}$, respectively. We take the initial perturbation amplitudes as $a_{n}(0)=R_{0} / 40 \mathrm{~cm}$, and $a_{2 n}(0)=0$. All patterns shown in Fig. 2 are plotted for $0 \leqslant t \leqslant 3 \mathrm{~s}$, in equal time intervals $\Delta t=0.3 \mathrm{~s}$.

By inspecting Figs. 2(a) and 2(b) it is evident that the purely linear calculation just leads to the evolution of slightly deformed, almost circular interfaces. It is true that despite of the fact that $A=0$, the elastic nature of the fluid-fluid boundary and the existence of a curvature-dependent bending rigidity allow interface destabilization. However, the linear destabilizing effects seem very modest. Moreover, regardless of the value of the initial radius $R_{0}$, the overall shapes of the patterns illustrated in Figs. 2(a) and 2(b) are quite similar.
A considerably different situation is depicted in Figs. 2(c) and 2(d) which exhibit the weakly nonlinear time evolution of the interfaces. First, it is clear that the resulting weakly nonlinear interfaces are considerably more deformed than their purely linear counterparts shown in Figs. 2(a) and 2(b). This indicates the importance of the nonlinear effects in providing sizable interface deformations already at the onset of nonlinearity. Moreover, notice that the morphologies of the resulting patterns depicted in Figs. 2(c) and 2(d) are significantly different from one another: while finger tipnarrowing is obtained in (c), finger tip-widening is the dominant phenomenon in (d). Since the only difference between Figs. 2(c) and 2(d) resides on the value of the initial radius $R_{0}$, this points to a strong sensitivity of the system to small changes in the initial conditions. So, a small initial difference in $R_{0}$ could be determinant in influencing the ultimate shape of the elastic interface patterns, which can present either wide or narrow fingering structures.

It is worth pointing out that this very strong sensitive dependence on the value of $R_{0}$ is not encountered in the weakly nonlinear analysis of the classical Saffman-Taylor instability [8]. What makes the present system special is the presence of high-order exponents of the unperturbed interface radius $R\left[R^{N}\right.$, with exponents $N$ as high as 12] that appear in the denominators of the expressions for the growth rate, and for the second-order mode-coupling function $F(n, m)$ [see Eqs. (9)-(12)]. The dependence on $R_{0}$ becomes even stronger because of the presence of the exponential term $e^{-\eta}$, where $\eta=(\lambda / R)^{2}$, in Eqs. (10)-(12). Therefore, tiny changes in $R_{0}$ can lead to significant changes in the nonlinear dynamics. This specific effect is considerably smaller in the classical Saffman-Taylor where the highest value for the exponent $N$ mentioned above is 4 , and where the exponential factor $e^{-\eta}$ is absent (see, e.g., Eqs. (19)-(21) in Ref. [8]).

Another interesting aspect of the weakly nonlinear dynamics can be unveiled by examining Fig. 3 which plots the time evolution of the first harmonic perturbation amplitude $a_{2 n}$ for (a) $R_{0}=1.2 \mathrm{~cm}$ and (b) $R_{0}=1.145 \mathrm{~cm}$, for different values of the rigidity fraction $C$. All other physical parameters and initial conditions are the same as the ones used in Fig. 2. From Fig. 3(a) we see that no matter what value of $C$ is used, the perturbation amplitude is always driven positive. Moreover, we notice that by increasing the value of $C$ one obtains increasingly larger magnitudes for $a_{2 n}>0$. In other words, as $C$ is increased one detects the occurrence of finger tip-narrowing events, presenting increasingly sharper finger tips. Within the scope of our perturbative mode-coupling theory, the sharpest finger situation occurs when $C=0.99$ that corresponds to the pattern illustrated in Fig. 2(c).

A distinct type of behavior is verified in Fig. 3(b): by increasing the magnitude $C$ from 0.70 to 0.90 one does observe the production of patterns having sharper finger tips. Nevertheless, for $C=0.99$ we verify that the perturbation amplitude $a_{2 n}$ goes strongly negative, indicating the formation of wider finger tips. This last situation corresponds to the pattern plotted in Fig. 2(d). So, Fig. 3(b) predicts the possible occurrence of a peculiar finger shape transition (from narrow to wide fingers) regulated by the tuning of the rigidity fraction parameter $C$. A possible physical explanation for the flattening of the fingers can be related to the fact that the own elastic nature of the 
(a)

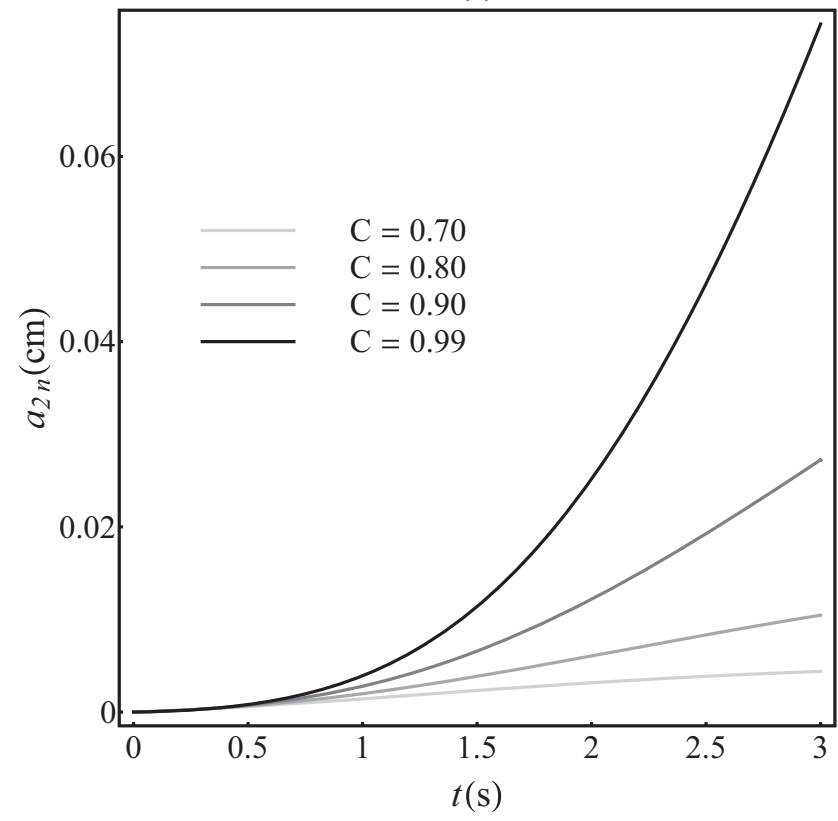

(b)

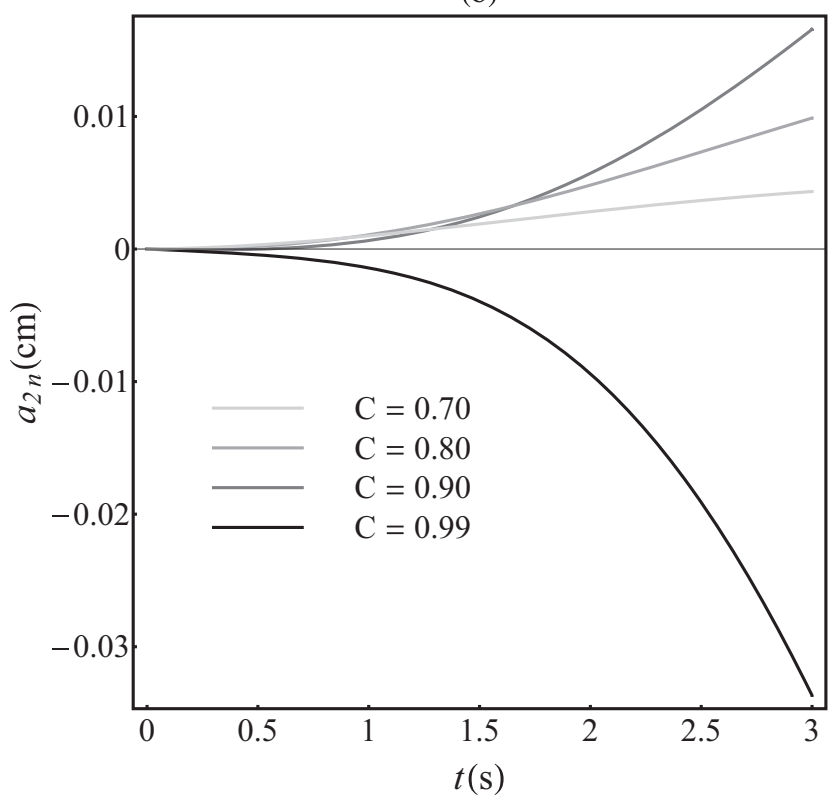

FIG. 3. Weakly nonlinear time evolution of the cosine perturbation amplitude $a_{2 n}$ for different values of $C$ when (a) $R_{0}=1.2 \mathrm{~cm}$ and (b) $R_{0}=1.145 \mathrm{~cm}$.

interface tends to inhibit the appearance of sharp structures. Therefore, the emergence of mushroom shapes or flat, wide fingers would be favored because each represent some approximation of a minimal curvature for the fluid-fluid interface.

We now turn our attention to the effect of the bending rigidity $v=v(\theta, t)$ while dynamically shaping the interface. Figure 4 displays the pattern formation of the bending rigidity field associated with the evolving interfaces portrayed in Fig. 2. The linear theory in Figs. 4(a) and 4(b) is characterized by smooth changes along the interfacial arc length with decreasing magnitude of $v$, centered at the maxima in curvature, while nearly straight regions present an effectively stiffer bending (a)

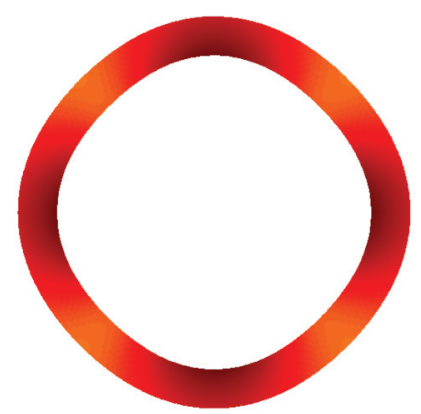

(c)

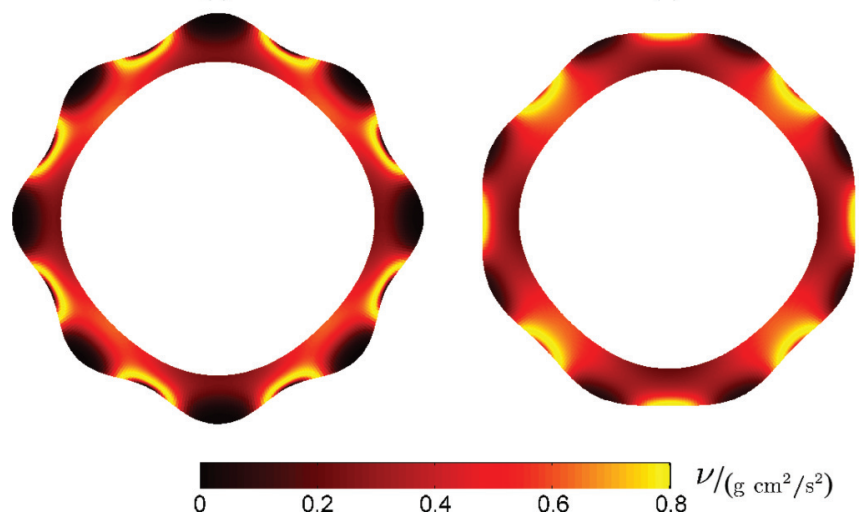

FIG. 4. (Color online) Time evolution of the bending rigidity field $v$ [Eq. (6)] in units of $\mathrm{g} \mathrm{cm}^{2} / \mathrm{s}^{2}$, for the evolving interfaces in Fig. 2. Purely linear evolution for (a) $R_{0}=1.2 \mathrm{~cm}$, and (b) $R_{0}=1.145 \mathrm{~cm}$, and weakly nonlinear evolution for (c) $R_{0}=1.2 \mathrm{~cm}$ and (d) $R_{0}=1.145 \mathrm{~cm}$. Note that smallest $v$ observed is 0.0013 .

elasticity. As the interface evolves, however, the initially perturbed bending rigidity distribution increases radially, with different rates, at every point of the interface. The radial growth of the interface reduces the overall curvature and, consequently, augments the magnitude of $v$, despite of the local elastic weakening effect from Eq. (6). This prediction is in agreement with the general behavior of the elastic resistance across the interface, which penalizes any increase in local curvature, given the energetically higher configuration. Nevertheless, as expected from the linear theory, Figs. 4(a) and 4(b) fail to provide the dynamical significance the elastic bending weakening behavior, given its intrinsic nonlinear contribution, Eq. (5). In this case, the bending rigidity $v$ modifies the elastic restoring forces dynamically, as it accounts for the fact that higher curvatures may brake the elastic binding between adjacent regions of the elastic interface, thus locally reducing the effective bending resistance and promoting fingering instability.

The weakly nonlinear solutions illustrated in Figs. 4(c) and 4(d) capture this complex interplay between the interface patterning and the bending rigidity field, compare for instance with Figs. 2(c) and 2(d). In this regime, nonlinearities are responsible for a new dynamical bifurcation at the level of the bending rigidity field $v(\theta, t)$, which causes the branching of the bending rigidity magnitude in time. The bending rigidity bifurcation may occur for higher [Fig. 4(c)] or lower [Fig. 4(d)] values of $\nu$. This is due to the fact that the bending rigidity modulation does not privilege outward or inward 
fingers, since $v$ does not depend on the sign of the local curvature. The competing effects between the effectively stiff and flexible regions along the evolving interface dynamically shift initially stable (unstable) regions to lower (higher) interfacial rigidities, shaping in this way the observed fingering pattern in Figs. 2(c) and 2(d).

\section{CONCLUDING REMARKS}

During the past few years there has been a considerable interest in the study of the so-called reactive Hele-Shaw flows. In this type of confined flow displacements the already interesting features of traditional viscous fingering phenomena $[1,2]$ are supplemented by the occurrence of chemical reactions at the fluid-fluid interface [21-29].

One particularly interesting experimental investigation on reactive Hele-Shaw flows has been carried out by Podgorski et al. [27], who studied the development of visually striking patterns (mushroom-shaped and tentacle-like structures) which arise due to chemical reactions at the boundary separating two miscible fluids of equal viscosity. The reaction induces the formation of a flexible gel-like layer between the fluids, that restrains further mixing, so that the interfacial instabilities are triggered by the own elastic nature of the interface. One noteworthy feature of this pattern-forming system is the fact that it characterizes the realization of a nonviscous elastic fingering instability. Here the classic Saffman-Taylor instability is not active due to the zero viscosity contrast between the fluids.

Recently, a theoretical model has been proposed [28,29] to explain the occurrence of instability between viscositymatched fluids as experimentally observed in Ref. [27]. By assuming that the fluid-fluid interface acts as an elastic membrane which has a curvature-dependent bending rigidity, He et al. [28,29] developed a linear stability calculation and showed that the interface can indeed become unstable, even under zero viscosity contrast circumstances. Note that this model refers to an elastic fingering instability arising at the fluid-fluid interface [27], which differs from an equally interesting elastic instability that also takes place in Hele-Shaw cells [30], but that originates within the bulk of a gel.

In this work, we revisited the interfacial elastic fingering problem originally studied in [28,29], and went beyond the purely linear regime by focusing our attention on the weakly nonlinear stages of the dynamics. This has been accomplished by employing an analytic mode-coupling approach that is valid at lowest nonlinear perturbative order [8]. This theoretical scheme allows one to study the influence of the elastic character of the interface on key morphological features of the resulting patterned finger-like structures. In this context, we have shown that nonlinear effects are essential to induce sizable deformations, as well as to determine the ultimate shape of the fingers. Specifically speaking, we have found that by properly tuning the rigidity fraction parameter $C$ and the initial interface radius $R_{0}$ one can get the prevalence of either finger tip-widening or finger tip-narrowing events. Finally, we verified that these basic morphological aspects are consistent with the curvature weakening effect discussed in Refs. [28,29], in the sense that interfacial elastic fingers arise more easily in regions of lower rigidity.

\section{ACKNOWLEDGMENTS}

J.A.M. and G.D.C. thank CNPq for financial support through the program "Instituto Nacional de Ciência e Tecnologia de Fluidos Complexos (INCT-FCx)", and FACEPE through PRONEM project No. APQ-1415-1.05/10. H.G. acknowledges support through an Oxford University Hooke Fellowship and is supported by Award KUK-C1-013-04 from King Abdullah University of Science and Technology.

\section{APPENDIX A: DERIVATION OF EQ. (5)}

In this appendix we derive the pressure jump condition in Eq. (5) associated with the presence of an interfacial elastic membrane, which effectively divides the fluid domain in two, by accounting for variations of the metric and curvature tensor describing the membrane surface [31]. The formalism presented here is general and independent of the coordinate system, and does not require specification of the coordinate components [29].

When the flexible membrane is away from the equilibrium configuration, it generates an elastic force density that is balanced by the viscous stresses across the interface [31]. We consider a Helfrich elastic membrane [32] with bending energy

$$
E=2 \int v(H) H^{2} d S,
$$

and membrane bending rigidity, $v(H)$, in units of energy, which may also be a function of the local mean curvature $H$, where $d S$ is the element of the membrane surface area. Equation (A1) neglects both the potential for spontaneous and Gaussian curvature, as well as membrane surface tension [31,32]. The mean curvature in Eq. (A1) measures how the membrane bends in space, and is given by the average of the sum of the two principal curvatures, along two independent directions at the membrane surface, defined by the tangent vectors $\mathbf{r}_{i} \equiv \partial_{i} \mathbf{r}$ with $i=\alpha, \beta$, where $\mathbf{r}(\alpha, \beta)$ is the instantaneous membrane configuration parametrized by internal coordinates $(\alpha, \beta)$. The surface area element $d S$ can be written in terms of the metric tensor $g_{i j} \equiv \mathbf{r}_{i} \cdot \mathbf{r}_{j}$, via $d S=\sqrt{g} d \alpha d \beta$, where $g$ denote the determinant of $g_{i j}$. Hence, the force per unit area associated with the membrane bending energy in Eq. (A1) can be computed through the variational derivative

$$
\mathbf{f}=-\frac{1}{\sqrt{g}} \frac{\delta E}{\delta \mathbf{r}} .
$$

For simplicity, we neglect the influence of nonzero curvature in the direction perpendicular to the plane of flow $(z-$ direction) [8], and consider a cylindrical membrane deforming parallel to the Hele-Shall plates, as represented schematically in Fig. 1. Under these conditions, the variation of the elastic bending energy simply reads

$$
\begin{aligned}
\delta E= & \int d \alpha d \beta\left\{-\left(\frac{3}{2} v \kappa^{2}+v^{\prime} \kappa^{3}\right) \hat{\mathbf{s}} \cdot \delta \mathbf{r}_{\alpha}\right. \\
& \left.+\left(v \kappa+\frac{v^{\prime} \kappa^{2}}{2}\right)\left[\frac{\hat{\mathbf{n}} \cdot \delta \mathbf{r}_{\alpha \alpha}}{\sqrt{\mathbf{r}_{\alpha} \cdot \mathbf{r}_{\alpha}}}-\frac{\left(\mathbf{r}_{\alpha \alpha} \cdot \mathbf{r}_{\alpha}\right) \hat{\mathbf{n}} \cdot \delta \mathbf{r}_{\alpha}}{\left(\mathbf{r}_{\alpha} \cdot \mathbf{r}_{\alpha}\right)^{\frac{3}{2}}}\right]\right\},
\end{aligned}
$$

where $\kappa$ is the interfacial curvature in the plane of flow, $\{\hat{\mathbf{s}}, \hat{\mathbf{n}}\}$ is the orthonormal Frenet basis, respectively, in terms of the 
tangent and normal vectors along the interfacial membrane, $\alpha$ parametrizes the arc length $s$ of the elastic interface, and the bending rigidity $v$ is a function of $\kappa$. We denote partial derivatives relative to the curvature $\kappa$ by primes, while subscripts represent derivatives relative to the parameter $\alpha$. Integration by parts of Eq. (A3) leads to boundary terms that do not contribute to the force distribution along the elastic interface. Finally, from Eq. (A2) the resulting force per unit area assumes a compact form

$$
\mathbf{f}=-\left[\partial_{s s}\left(\nu \kappa+\frac{\nu^{\prime} \kappa^{2}}{2}\right)+\frac{1}{2}\left(\nu \kappa^{3}+v^{\prime} \kappa^{4}\right)\right] \hat{\mathbf{n}},
$$

with a vanishing contribution of the force on the tangential direction. This follows from the fact that the bending energy of a cylindrical elastic membrane is invariant under changes in coordinates, and therefore it does not vary under deformations at every point along the tangential direction $\hat{\mathbf{s}}$, despite $v=$ $\nu(\kappa)$. Furthermore, Eq. (A4) agrees with the result reported in Refs. [28,29]. The curvature, however, presented in the latter must be interpreted as the interfacial curvature, instead of mean curvature as argued in the text of the Refs. [28,29]. Multiple factors of two would be introduced in Eq. (A4) if rewritten in terms of the mean curvature [31].

The balance of the membrane elastic force density Eq. (A4) and the local stresses across the membrane surface, results on the modified Young-Laplace equation for an elastic interface

$$
\hat{\mathbf{n}} \cdot\left[\boldsymbol{\pi}^{(2)}-\boldsymbol{\pi}^{(1)}\right] \cdot \hat{\mathbf{n}}=\mathbf{f},
$$

where $\pi$

$$
\pi_{i k}=-p \delta_{i k}+\eta\left[\frac{\partial v_{i}}{\partial x_{k}}+\frac{\partial v_{k}}{\partial x_{i}}\right]
$$

is the local stress tensor representing both normal and viscous stresses in the fluid. Equation (A6) includes a viscous friction term proportional to the fluid's viscosity $\eta, \delta_{i k}$ denotes the Kronecker $\delta$ function, and $v_{i}$ represents the $i$ th component of the fluids' velocity vector. The pressure condition presented in the main text Eq. (5) is then obtained by matching the diagonal terms in Eq. (A5). It is worth noting, however, that, in general, viscous stresses originating from normal velocity gradients may also contribute for the radial geometry via Eq. (A5), as previously studied in [33].

\section{APPENDIX B: FUNCTIONS APPEARING IN THE MODE-COUPLING TERM $F(n, m)$}

This appendix presents the expressions for the functions $B_{1}(n, m), B_{2}(n, m), B_{3}(n, m)$, and $B_{4}(n, m)$ which appear in Eq. (12) of the text

$$
\begin{aligned}
B_{1}(n, m)= & -3+\frac{15}{4} m(n-m)+10(n-m)^{2}-\frac{9}{2} m^{2}(n-m)^{2} \\
& -6 m(n-m)^{3}-4(n-m)^{4}, \\
B_{2}(n, m)= & \frac{39}{2}-30 m(n-m)-71(n-m)^{2} \\
& +\frac{81}{2} m^{2}(n-m)^{2}+54 m(n-m)^{3}+32(n-m)^{4} \\
& -12 m^{2}(n-m)^{4}-12 m^{3}(n-m)^{3}, \\
B_{3}(n, m)= & -14+25 m(n-m)+54(n-m)^{2} \\
& -36 m^{2}(n-m)^{2}-48 m(n-m)^{3}-26(n-m)^{4} \\
& +18 m^{2}(n-m)^{4}+18 m^{3}(n-m)^{3},
\end{aligned}
$$

and

$$
\begin{aligned}
B_{4}(n, m)= & 1-2 m(n-m)-4(n-m)^{2} \\
& +3 m^{2}(n-m)^{2}+4 m(n-m)^{3}+2(n-m)^{4} \\
& -2 m^{2}(n-m)^{4}-2 m^{3}(n-m)^{3} .
\end{aligned}
$$

[1] P. G. Saffman and G. I. Taylor, Proc. R. Soc. London Ser. A 245, 312 (1958).

[2] For review papers, see G. M. Homsy, Annu. Rev. Fluid Mech. 19, 271 (1987); K. V. McCloud and J. V. Maher, Phys. Rep. 260, 139 (1995); J. Casademunt, Chaos 14, 809 (2004).

[3] L. Paterson, J. Fluid Mech. 113, 513 (1981).

[4] H. Thomé, M. Rabaud, V. Hakim, and Y. Couder, Phys. Fluids A 1, 224 (1989).

[5] J.-D. Chen, J. Fluid Mech. 201, 223 (1989); Exp. Fluids 5, 363 (1987).

[6] S. S. S. Cardoso and A. W. Woods, J. Fluid Mech. 289, 351 (1995).

[7] O. Praud and H. L. Swinney, Phys. Rev. E 72, 011406 (2005).

[8] J. A. Miranda and M. Widom, Physica D 120, 315 (1998).

[9] S. W. Li, J. S. Lowengrub, and P. H. Leo, J. Comput. Phys. 225, 554 (2007).

[10] B. Jha, L. Cueto-Felgueroso, and R. Juanes, Phys. Rev. Lett. 106, 194502 (2011)

[11] T. T. Al-Housseiny, P. A. Tsai, and H. A. Stone, Nat. Phys. 8, 747 (2012).
[12] T. T. Al-Housseiny and H. A. Stone, Phys. Fluids 25, 092102 (2013).

[13] R. M. Wilson, Phys. Today 65(10), 15 (2012).

[14] H. Guo, D. C. Hong, and D. A. Kurtze, Phys. Rev. Lett. 69, 1520 (1992).

[15] H. Guo, D. C. Hong, and D. A. Kurtze, Phys. Rev. E 51, 4469 (1995).

[16] C. K. Chan and N. Y. Liang, Phys. Rev. Lett. 79, 4381 (1997).

[17] C. K. Chan, Physica A 288, 315 (2000).

[18] R. Krechetnikov and G. M. Homsy, J. Fluid Mech. 509, 103 (2004).

[19] J. Fernandez, R. Krechetnikov, and G. M. Homsy, J. Fluid Mech. 527, 197 (2005).

[20] F. M. Rocha and J. A. Miranda, Phys. Rev. E 87, 013017 (2013).

[21] A. De Wit, K. Eckert, and S. Kalliadasis, Chaos 22, 037101 (2012).

[22] C. Almarcha, P. M. J. Trevelyan, P. Grosfils, and A. De Wit, Phys. Rev. Lett. 104, 044501 (2010).

[23] M. Mishra, P. M. J. Trevelyan, C. Almarcha, and A. De Wit, Phys. Rev. Lett. 105, 204501 (2010). 
[24] L. A. Riolfo, Y. Nagatsu, S. Iwata, R. Maes, P. M. J. Trevelyan, and A. De Wit, Phys. Rev. E 85, 015304(R) (2012).

[25] L. A. Riolfo, J. Carballido-Landeira, C. O. Bounds, J. A. Pojman, S. Kalliadasis, and A. De Wit, Chem. Phys. Lett. 534, 13 (2012).

[26] A. R. White and T. Ward, Chaos 22, 037114 (2012).

[27] T. Podgorski, M. C. Sostarecz, S. Zorman, and A. Belmonte, Phys. Rev. E 76, 016202 (2007).
[28] A. He, J. S. Lowengrub, and A. Belmonte, SIAM J. Appl. Math. 72, 842 (2012).

[29] A. He, Ph.D. thesis, Penn State University (2011).

[30] B. Saintyves, O. Dauchot, and E. Bouchaud, Phys. Rev. Lett. 111, 047801 (2013).

[31] T. R. Powers, Rev. Mod. Phys. 82, 1607 (2010).

[32] Ou-Yang Zhong-can and W. Helfrich, Phys. Rev. Lett. 59, 2486 (1987).

[33] H. Gadêlha and J. A. Miranda, Phys. Rev. E 79, 066312 (2009). 